\title{
Ações de Ciência, Tecnologia e Inovação em Saúde para a Amazônia Legal
}

Correspondência | Correspondence: Decit - Departamento de Ciência e Tecnologia do Ministério da Saúde Esplanada dos Ministérios

Bloco $G$ sala 845

70058-900 Brasília, DF, Brasil

Texto de difusão técnico-científica do Ministério de Saúde.

\section{Science, Technology and Health Innovation actions in the Legal Amazon of Brazil}

\author{
Departamento de Ciência e Tecnologia, Secretaria de Ciência, \\ Tecnologia e Insumos Estratégicos, Ministério da Saúde
}

\begin{abstract}
A Amazônia desperta interesses nacionais e internacionais devido à importância das questões referentes à diversidade biológica, ao clima e à água. $\mathrm{O}$ estabelecimento da definição geopolítica "Amazônia Legal", em 1966, limitou a região em nove Estados (Acre, Amapá, Amazonas, oeste do Maranhão, norte do Mato Grosso, Pará, Rondônia, Roraima e Tocantins). Compõe-se de 808 municípios distribuídos em uma área que compreende cerca de $61 \%$ do território nacional.* A extensa fronteira que essa região faz com sete países concentra: a maior diversidade social do planeta; a mais exuberante e complexa biodiversidade mundial; $30 \%$ do estoque genético da Terra; um terço das florestas do planeta; a maior bacia hidrográfica, o maior rio em volume de água; um quinto das reservas hídricas do planeta e a maior província mineral mundial.**
\end{abstract}

A riqueza dessas características e a importância estratégica da atração de capitais para o desenvolvimento de atividades econômicas já era reconhecida na segunda metade do século XVIII. Desde então, os governantes sabiam que apenas as demarcações do território não bastavam, era necessário se fazer presente para aproveitar melhor as riquezas da região. Destacase a avidez européia em usufruir das riquezas da Amazônia sem resultar em nenhum desenvolvimento lo- cal. *** A partir desse período até meados da década de 1940, passando pelo auge do crescimento econômico ocasionado pela exploração da borracha, as políticas para a região tiveram caráter basicamente colonialista e não visaram o desenvolvimento econômico e social.****

As décadas seguintes foram marcadas por políticas que, embora buscassem o desenvolvimento regional, voltaram-se, basicamente, para a exploração das potencialidades locais ou para ações compensatórias a fim de abrandar as desigualdades regionais.***** Os reflexos do atraso no desenvolvimento em relação às outras regiões do País podem ser verificados em análise do Índice de Desenvolvimento Humano (IDH) preconizado pelo Programa das Nações Unidas para o Desenvolvimento. Para os nove Estados que compõem a Amazônia Legal, o Mato Grosso é o Estado com a melhor classificação quanto ao IDH, ficando o Maranhão com a última colocação no ranking. ******

A partir da década de 1950, políticas para planejamento e promoção de C\&T começaram a ser formuladas e implantadas no País.******* Os investimentos públicos para a região se voltaram para ações capazes de gerar desenvolvimento, tais como criação de institu-

\footnotetext{
*Ministério da Saúde, Departamento de Apoio à Descentralização. Plano de qualificação da atenção à saúde na Amazônia Legal: saúde Amazônia. Brasília (DF); 2006. (Série C - Projetos, Programas e Relatórios).

**M Ministério da Saúde, Secretaria-Executiva, Departamento de Apoio à Descentralização. Saúde Amazônia: relato de processo, pressupostos, diretrizes e perspectivas de trabalho para 2004. Brasília (DF); 2003. (Série C - Projetos, Programas e Relatórios).

***Val AL. Formação e fixação de recursos humanos: ações essenciais para a Amazônia. Ciênc Cult (São Paulo). 2006;58(3):79-86.

****Ministério da Saúde, Secretaria de Ciência, Tecnologia e Insumos Estratégicos, Departamento de Ciência e Tecnologia. Proposta de atuação em ciência e tecnologia em saúde na Amazônia Legal. Brasília (DF); 2004.

*****Barros FAF. Os desequilíbrios regionais da produção técnico-científica. São Paulo Perspect. 2000;14(3):12-9.

******Programa das N ações Unidas para o Desenvolvimento. Disponível em http:// www.pnud.org.br/atlas/ranking [acesso em 23 out 2006]

********Conde MVF, Araújo-Jorge TC. Modelos e concepções de inovação: a transição de paradigmas, a reforma da C\&T brasileira e as concepções de gestores de uma instituição pública de pesquisa em saúde. Ciênc Saúde Coletiva. 2003;8(3):727-41.
} 


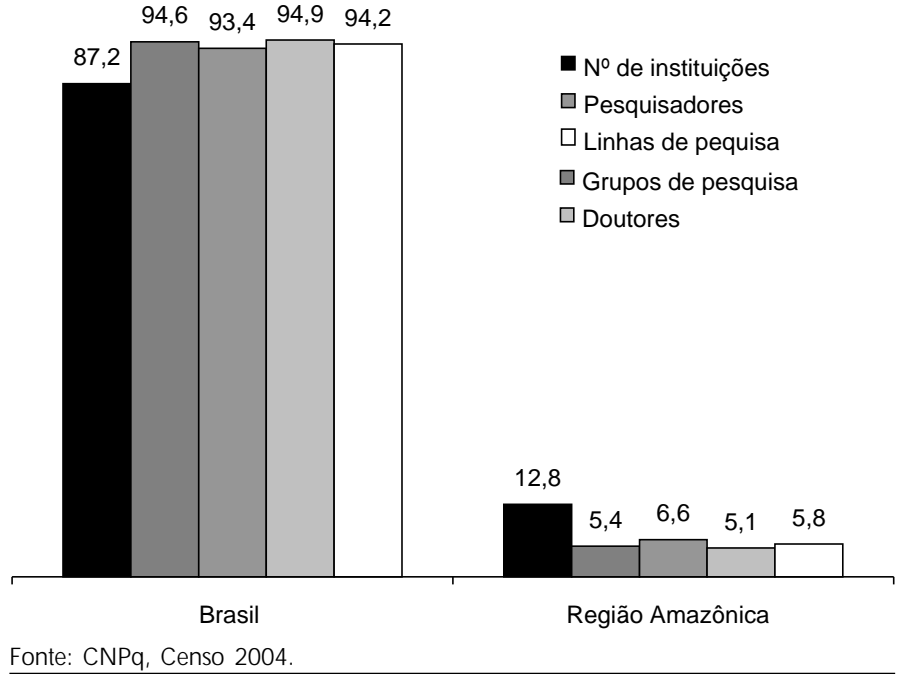

Figura 1 - Distribuição do percentual de instituições, grupos de pesquisa, pesquisadores, doutores e linhas de pesquisa, para todas as áreas do conhecimento, segundo região amazônica e Brasil. levou o governo federal a considerar a região como prioridade e a lançar, em 2003, o Programa de Desenvolvimento Sustentável da Amazônia. Esse Programa reuniu esforços dos ministérios, gestores locais, controle social, representantes da sociedade civil e universidades para definir ações prioritárias para a região. Os seguintes temas foram abordados: produção sustentável com tecnologia avançada, novo padrão de financiamento, gestão ambiental e ordenamento territorial, infra-estrutura para o desenvolvimento e inclusão social e cidadania. Dentro desse último tema, o Ministério da Saúde iniciou, por meio de um grupo de trabalho, a discussão da temática saúde que resultou na realização de algumas oficinas de trabalho locais. tos de pesquisa, universidades e grandes obras de infraestrutura.* No entanto, todas essas transformações e o conseqüente crescimento econômico estão aquém das demais regiões do País, pois não foram acompanhadas por políticas de fixação e capacitação de recursos humanos, essenciais para a manutenção da cadeia de desenvolvimento econômico e social.

Há carência de investimentos para a consolidação de uma base técnico-científica, capaz de participar ativamente do processo de desenvolvimento local. Os efeitos dessa carência podem ser observados ao comparar a distribuição dos grupos de pesquisa, pesquisadores, número de mestres, doutores e programas de pós-graduação, para todas as áreas do conhecimento na região amazônica com os outros Estados (Figura 1).

\section{Políticas atuais}

Os primeiros anos do século XXI marcam uma fase de convergência de esforços políticos para a consolidação do desenvolvimento da região. Exemplos recentes são a política dos Fundos Setoriais, o Programa Acelera Amazônia, iniciativa da Coordenação de Aperfeiçoamento de Pessoal de Nível Superior, e o envolvimento da Sociedade Brasileira para o Progresso da Ciência na articulação de uma agenda de C\&T para a região*

A permanência de uma situação de desigualdade
A primeira oficina, realizada em Manaus, AM, em 2003, objetivou identificar os problemas e dificuldades no campo da atenção à saúde, da gestão do trabalho e do financiamento de Ciência, Tecnologia e Inovação em Saúde (CT\&I/S). Identificada a problemática da $\mathrm{CT} \& \mathrm{I} / \mathrm{S}$ na região e com o intuito de consolidar propostas na área de pesquisa, desenvolvimento e formação de recursos humanos, foram realizadas outras três oficinas locais, sob a orientação do Ministério da Saúde, nos anos de 2003 e 2004.** Os resultados dessas of icinas foram:

- formação do Acordo Multilateral de Cooperação Técnico Científica em Saúde das Instituições da Amazônia: liderado pela Fundação Oswaldo Cruz, tem como objetivos a construção de uma rede cooperativa de pesquisas, formação de recursos humanos, cooperação técnica com os gestores do Sistema Único de Saúde (SUS) e cooperação internacional, envolvendo as instituições de ciência e tecnologia da região; $* * *$

- estabelecimento de sete áreas prioritárias de pesquisa, contempladas em editais lançados pelo Ministério da Saúde: malária, micobacterioses, síndromes febris ictero-hemorrágicas agudas, avaliação de programas e de serviços em saúde, saúde e ambiente, nutrição e alimentação e fitoterápicos;

- implementação de um curso de mestrado na área de "Saúde, Sociedade e Endemias" e um curso de doutorado em Saúde Coletiva, coordenados pela Fundação Oswaldo Cruz, Universidade Federal do Amazonas e Universidade Federal do Pará.

*Val AL. Formação e fixação de recursos humanos: ações essenciais para a Amazônia. Ciênc Cult (São Paulo). 2006;58(3):79-86.

**Ministério da Saúde, Secretaria de Ciência, Tecnologia e Insumos Estratégicos, Departamento de Ciência e Tecnologia. Relatório das ações do Departamento de Ciência e Tecnologia na região amazônica. Brasília (DF); 2006.

***Relatório de Atividades do Acordo Multilateral de Cooperação Técnico-Científica em Saúde nas Instituições da Amazônia. Brasília (DF); 2005. 


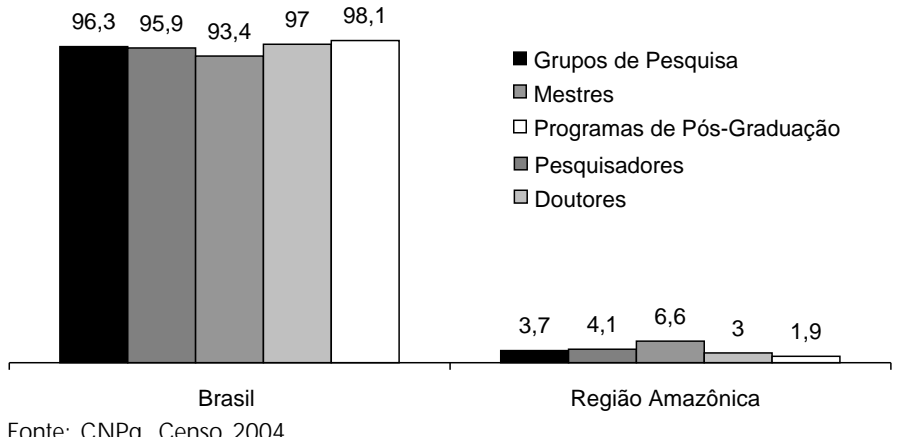

NPq, Censo 2004

Figura 2 - Distribuição percentual de grupos de pesquisa, pesquisadores, mestres, doutores e programas de pós-graduação, para a grande área de ciências da saúde, segundo região amazônica e Brasil.

\section{Ações do Departamento de Ciência e Tecnologia para a região da Amazônia Legal}

As desigualdades socioeconômicas da região condicionam os desequilíbrios nos indicadores de infra-estrutura e de recursos humanos em CT\&I/S, dificultando a captação de investimentos para essa área, sabidamente concentrados nas regiões Sudeste e Sul (Figura 2).

Como forma de minimizar essas desigualdades, o Ministério da Saúde, por meio do Departamento de Ciência e Tecnologia (Decit) da Secretaria de Ciência, Tecnologia e Insumos Estratégicos, vem priorizando a região amazônica com programas que visam fortalecer o esforço local em CT\&I/S. Dentre as diversas ações executadas, desde $2000,{ }^{*}$ destaca-se o Programa Pesquisa para o SUS/ Saúde Amazônia (PPSUS/Saúde Amazônia), componente, para a região, do Programa Pesquisa para o SUS: gestão compartilhada em Saúde (PPSUS).

O PPSUS é uma estratégia de fomento descentralizado à pesquisa em saúde, conduzida pelo Decit em parceria com Fundações de Amparo à Pesquisa (FAP), Secretarias Estaduais de Saúde (SES), Secretarias Estaduais de Ciência e Tecnologia (SCT) e o Conselho Nacional de Desenvolvimento Científico e Tecnológico $(\mathrm{CNPq})$. Esse programa visa apoiar o desenvolvimento de pesquisas que contribuam para a compreensão e resolução de problemas prioritários de saúde nos Estados, fortalecer a gestão local de CT\&I/S e reduzir as desigualdades nessa área.**

O campo de ação do PPSUS/Saúde Amazônia compreende o apoio financeiro a pesquisas

Fonte: Decit, 2006 investidos. em saúde, por meio da publicação de editais, a cinco Estados da região: Acre, Amapá, Rondônia, Roraima e Tocantins. Esses Estados apresentam os menores indicadores de $\mathrm{CT \& I} / \mathrm{S}$ e à época, não contavam com instâncias formais de fomento à pesquisa para a realização de parcerias. Os temas de pesquisa dos editais foram os definidos nas oficinas de trabalho locais, conduzidas pelo Ministério da Saúde e com a participação de gestores de saúde e representantes da comunidade científica. Além disso, foram consideradas as vocações dos grupos de pesquisa locais.

Foram lançados editais, um no ano de 2004 e outro no ano de 2005, para os cinco Estados já citados, com investimento global de $\mathrm{R} \$ 1,6$ milhões e 29 projetos de pesquisa financiados (Figura 3).

Os Estados do Amazonas, Maranhão, Mato Grosso e Pará, com instâncias de fomento à pesquisa consolidadas, participaram como parceiros do Decit na condução estadual do PPSUS. Foram lançados editais para financiamento de pesquisa nos quatro Estados, nos anos de 2004 e 2005 , e investidos cerca de $\mathrm{R} \$ 2,3$ milhões em 55 projetos de pesquisa (Figura 4).

A inclusão de quatro Estados da região (Acre, Amapá, Roraima e Tocantins) ao PPSUS, no ano de 2006, aumenta o volume de recursos a serem investidos em pesquisa para esse ano, atingindo cerca de $\mathrm{R} \$ 5$ milhões.

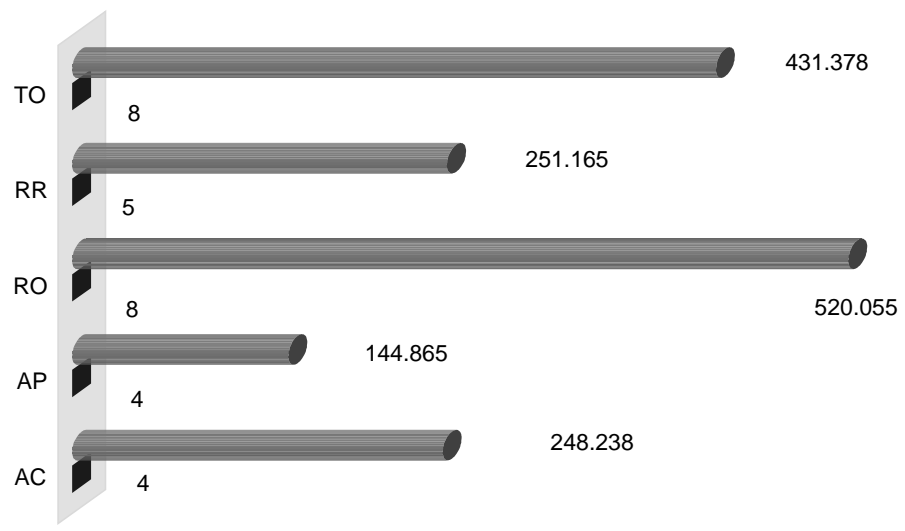

- Número de projetos Valor Investido (em mil reais)

Figura 3 - Distribuição do número de pesquisa apoiadas pelo PPSUS/ Saúde Amazônia, nos anos de 2004 e 2005, segundo UF e valores

*Ministério da Saúde, Secretaria de Ciência, Tecnologia e Insumos Estratégicos, Departamento de Ciência e Tecnologia. Proposta de atuação em ciência e tecnologia em saúde na Amazônia Legal. Brasília (DF); 2004.

**M inistério da Saúde, Secretaria de Ciência, Tecnologia e Insumos Estratégicos, Departamento de Ciência e Tecnologia. Diretrizes técnicas do programa pesquisa para o SUS: gestão compartilhada em saúde. 2ª ed rev. Brasília (DF); 2006. (Série A - Normas e Manuais Técnicos). 


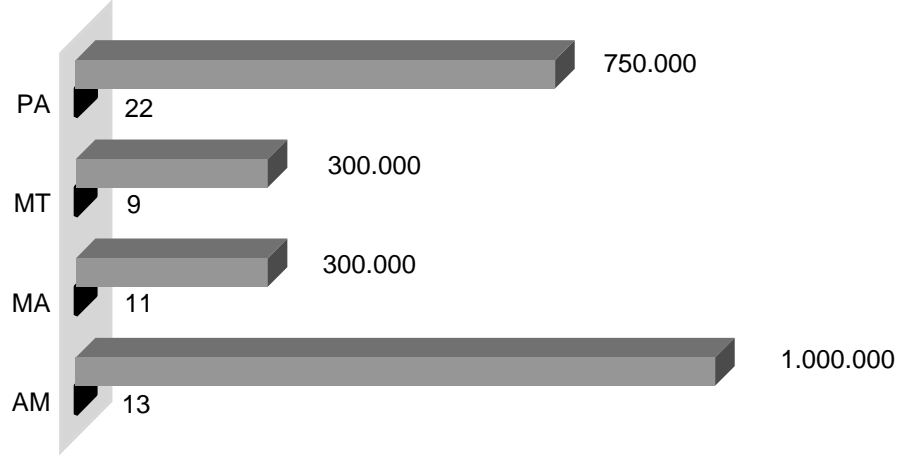

Número de projetos $\quad$ Valor (em mil reais)

Fonte: Decit, 2006.

Figura 4 - Distribuição do número de pesquisas apoiadas pelo PPSUS em 2005, na Amazônia Legal, segundo UF e valores investidos. direcionado para financiar propostas de ações no campo da saúde especificamente para a região da BR-163, importante rodovia que se estende pela região amazônica.

Os esforços foram empreendidos pelo Decit no sentido de fortalecer o desenvolvimento de pesquisa na região, com conseqüente fortalecimento da base técnico-científica local e melhoria da situação de saúde. Tais esforços ainda englobam o apoio a sete pesquisas em temas considerados estratégicos (com investimentos de cerca de $\mathrm{R} \$ 3 \mathrm{mi}$ lhões); três novos centros de pesquisa clínica (R $\$ 3,8$ milhões); e 17 Comitês de Ética ( $\mathrm{R} \$ 4,4$ milhões).

\section{Editais temáticos}

O Decit possui outro mecanismo de fomento à pesquisa viabilizado pela publicação anual de editais temáticos, que apóiam financeiramente projetos de pesquisa e desenvolvimento tecnológico, em consonância com a Agenda Nacional de Prioridades de Pesquisa em Saúde (ANPPS). O público-alvo desses editais são universidades e instituições de pesquisa de todo o País, sendo que para os Estados da região amazônica foram selecionados 15 projetos no ano de 2004 e 22 em 2005. Embora esses resultados ainda apontem uma desigualdade na captação de recursos pela região, houve substancial evolução tanto no número de projetos como no valor dos financiamentos (Figura 5). Ressalta-se que, dentro dessa estratégia de fomento, um dos editais temáticos de 2005 foi

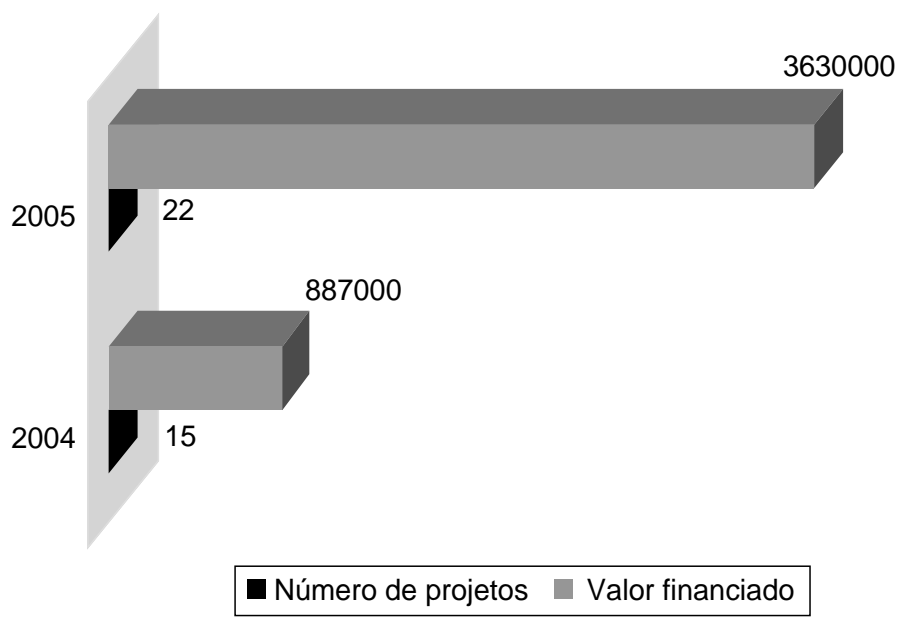

Fonte: Decit, 2006.

Figura 5 - Distribuição do número de pesquisas apoiadas e valores investidos pelos editais temáticos, na Amazônia Legal, segundo ano.

\section{CONCLUSÕES}

A constituição do Decit, em 2000, e a posterior criação da Secretaria de Ciência, Tecnologia e Insumos Estratégicos, em 2003, fazem parte importante do cenário de mudanças recentes ocorridas no campo da CT\&I/S. Destaque-se a realização da $2^{\mathrm{a}}$ Conferência Nacional de Ciência, Tecnologia e Inovação em Saúde e a aprovação da Agenda Nacional de Prioridades de Pesquisa em Saúde* (ANPPS) e da Política Nacional de Ciência, Tecnologia e Inovação em Saúde, em 2004. O direcionamento das ações do Decit para os Estados da Amazônia Legal, em particular o PPSUS/ Saúde Amazônia e o PPSUS, pretendem diminuir as desigualdades regionais no campo da CT\&I/S na medida em que promovem a produção de novos conhecimentos, contribuem para a consolidação de pesquisa, criação de novos grupos e, ainda, fortalecem o sistema local de ciência e tecnologia em saúde.

Os resultados alcançados por essa relativamente nova e relevante fonte de financiamentos podem ser observados, por exemplo, com a inclusão, no PPSUS, de quatro Estados da região que anteriormente estavam inseridos no PPSUS/Saúde Amazônia. Esses Estados, sem instância formal de ciência e tecnologia, ainda não podiam estabelecer convênio com o Decit/MS/CNPq. A formação dessas novas parcerias mostra $\mathrm{o}$ crescente reconhecimento por parte de SES e SCT, da importância da geração do conhecimento na cadeia de desenvolvimento social para a melhoria das condições de saúde da população.

*2a Conferência Nacional de Ciência, Tecnologia e Inovação em Saúde, Brasília, 25 a 28 de julho de 2004: Anais / Ministério da Saúde, Secretaria de Ciência, Tecnologia e Insumos Estratégicos, Departamento de Ciência e Tecnologia. Conselho Nacional de Saúde. Brasília (DF): Ministério da Saúde; 2005. p. 272. (Série D. Reuniões e Conferências). 
Espera-se ainda que os resultados dessas ações influenciem positivamente os indicadores de recursos humanos qualificados para a pesquisa. Isso porque muitas das pesquisas apoiadas, coordenadas por doutores que detêm expertise nas suas respectivas áreas, têm em suas equipes mestrandos e doutorandos beneficiados do desenvolvimento da pesquisa.

Outras carências no setor de CT\&I/S também pode- rão ser minimizadas com as iniciativas apresentadas; seja por meio da promoção de maior aproximação entre o sistema acadêmico e o sistema de gestão em saúde, da disseminação da produção científica entre gestores e outros atores envolvidos e da atenção dada às especificidades regionais e setoriais, todas proporcionando uma melhor distribuição dos recursos destinados à pesquisa em saúde, contribuindo para diminuir a iniqüidade no setor. 